\title{
Experimental Study on Free Vibratory Behavior of Nonlinear Structure
}

\author{
Denes Farago', Zoltan Dombovari ${ }^{1 *}$ \\ 1 Department of Applied Mechanics, Faculty of Mechanical Engineering, Budapest University of Technology and Economics, \\ H-1111 Budapest, Múegyetem rkp. 3., Hungary \\ *Corresponding author, e-mail: dombovari@mm.bme.hu
}

Received: 01 May 2018, Accepted: 17 January 2019, Published online: 25 March 2019

\begin{abstract}
The basic behavior of free vibratory nonlinear system is investigated in this work. The main approach was to reveal the order of fitting necessary to properly approximate the actual behavior of a nonlinear mechanical system. Tests were performed in an experimental setup subjected to geometric hardening nonlinearities. The investigation showed that, it is essential to approximate the nonlinearity correctly. Low order approximation can result in large errors in the predicted amplitudes. The nonlinear static stiffness characteristics was measured and fit with polynomial describing function. The free vibratory response was deviated from the one calculated by cubic fitting. The presented higher order approximation of the amplitude-frequency parametric relation is revealed so that, in this particular example, seventh degree approximation is sufficiently closer to the experienced behavior. The analytical solution including the first and second order internal resonances were checked and compared with continuation results of the backbone curve.
\end{abstract}

\section{Keywords}

nonlinear vibration, hardening spring, backbone curve

\section{Introduction}

The main motivation of this work is to have an experimental framework for validating possible future theoretical developments related to nonlinear structural dynamics. Nonlinearity, in general, influences the anyway standard modal characterization techniques resulting deviation in modeling and in predicting overall dynamic behavior of usually relatively large flexible structures. This is an important problem in airplane structure [1], in windmill pillars [2] or e.g. in machine tool dynamics [3, 4]. In many cases, the nonlinearities in question are weak, meaning that the natural frequencies and damping factors are not effected much. Rather, the reflected stiffness is affected through the concept of nonlinear normal modes (NNM) [5].

Although the example presented in the paper is considered as one degree of freedom (DoF) mechanical system, the measurement of free vibratory response is essential to characterize large amplitude behavior of multi-DoF systems, too. Here the free vibratory characteristics are measured and theoretically determined through the concept of backbone curve $[6,7]$, which is essentially a projection of NNM on the extended space with frequency.
In the literature, there are various attempts to build up a consistent framework for nonlinear mechanical systems similarly to the linear modal analysis. Rosenberg [8] has introduced the NNM's of multi-DoF nonlinear systems as their mode reach the maximum in all modal coordinates synchronously at the same time. The other, stricter definition is for conservative systems originated from Shaw and Pierre [9] who defined normal modes as invariant manifolds. Szalai et al. [10] was using the concept of smoothest invariant manifold to describe the behavior of NNM's. Kerschen et al. in [5] shows that non-conservative (damped) free systems are settled along the backbone curves, which is essentially the amplitude-frequency curves of the closed orbits on the invariant manifolds of the undamped systems.

Large, roughly monolithic structures can undergo elastic but large amplitude dynamic deformation, when the effect of geometric nonlinearities come to the front as nonlinearity in stiffness. Two basic stiffness characteristics can be distinguished, the so-called progressive characteristics (for hardening springs), and the degressive one (for softening 
springs). The most noticeable sign of nonlinear vibrations is that the (apparent) frequency of the free vibration depends on the amplitude. Therefore, the definition of the natural frequencies is changed to be strictly related to the characteristics of the local dynamic behavior on the linear subspace. The backbone curve (blue curve on Fig. 1) shows the relation between the amplitude and the frequency of the undamped free vibration of 1 DoF systems. The small amplitude vibration behaves as a simple linear one DoF system locally with linear stiffness, and its frequency is referred to as the natural angular frequency $\left(\omega_{\mathrm{n}}\right)$ even for the original nonlinear system. Forced, undamped one DoF vibrations have deviated "resonance" curves, which characteristics depend on the driving force level. This actually, in one sense, corresponds to the very definition of a nonlinear mechanical system. Moreover, multiple periodic solutions can coexist for a specific driving frequency (black on Fig. 1), where continuous black and dashed curves represent the stable and the unstable time periodic forced responses of the nonlinear system, respectively.

In this paper, we characterize the nonlinear system by measuring its nonlinear stiffness characteristics by performing free vibratory experiments. Then, by assuming one DoF nonlinear dynamics, we compare the theoretical calculation based on the static stiffness characteristics. The tests can be considered as a preliminary experimental characterization of a nonlinear mechanical test bench, with which nonlinear modal test will be carried out.

\section{Experiments}

The aim in this section was to see how accurate is the one DoF nonlinear model of the experimental setup considering its static stiffness nonlinearity and its linear modal behavior. The tests were carried out on a simple beam structure, similar to the one in [11], which utilizes the geometric nonlinearities of a slender fixed beam segment (Fig. 2).

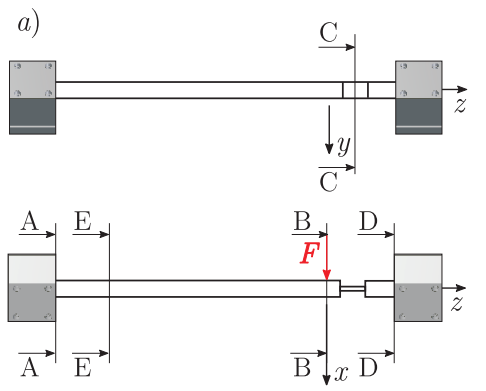

b)

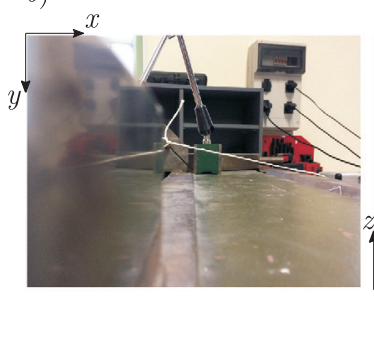

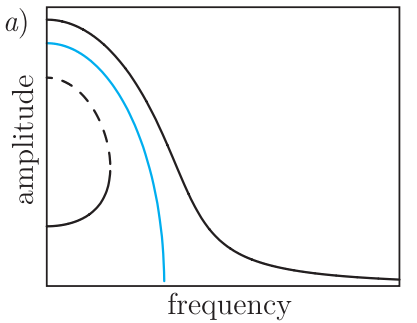

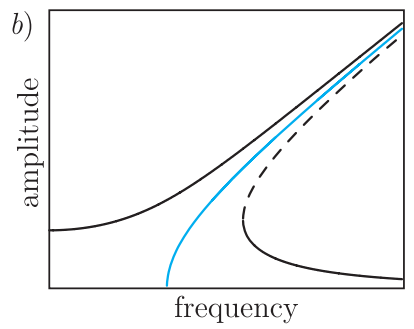

Fig. 1 Nonlinear undamped resonance curves (stable: continuous black, unstable: dashed black) and the corresponding backbone curves (blue) determined for softening (a), and with hardening (b) cases.

\subsection{Experimental structure}

A simple square cross section beam is built with special supports (Fig. 2 (b)) to introduce stiff almost ideally fixed constrains with insignificant introduced damping in both ends. The square beam segment is weakened by a short slender steel plate asymmetrically. This plate is fixed to the longer and the shorter part of the beam with square cross section (as shown on Fig. 2 (a), (b)). This structural weakening decreases the overall stiffness of the beam, and introduces nonlinear behavior. In this manner, larger displacements can be achieved ( $a$ few $\mathrm{mm}$ near the plate). The strain in the plate is large enough, to reach elastic nonlinear regions that cannot be neglected in the modeling. Using this elastic geometric nonlinearity phenomenon we introduce progressive hardening behavior in the mechanical system.

\subsection{Static characterization}

As seen on Fig. 2 (a) (b), the load is applied at the same cross section of the beam, where the displacement is measured at point B. In the experiment, the displacement was measured with $0.01 \mathrm{~mm}$ accuracy, and the load was achieved by hanging weights of different masses on a string attached to the beam (Fig. 2 (b)). The load was increased mostly with $10 \mathrm{~N}$ steps, up to $200 \mathrm{~N}$. The measurement was carried out both the positive and the negative

Fig. 2 In (a) the schematic representation of the nonlinear structure is presented. In (b) the circumstances of the static stiffness characterization at $\mathrm{B}-\mathrm{B}$ is presented, while the results are shown in (c) denoted by black dots along with a cubic polynomial fitting according to Eq. (1) (red line, $\epsilon$ is the relative error between the fit curve and the measurement). 
$x$ direction. This was necessary, because the actual structure had some slight asymmetries.

The direction of the load was not exactly parallel with the $x$ axis. This angle $(12.3 \mathrm{deg})$ was taken into consideration, when the force was calculated in the direction of the measured displacement.

As we can see on Fig. 2, the measurement points seemingly fit well on a 3rd order polynomial (see relative error $\epsilon$ ), however the coefficient of $x^{2}$ is quite large (in Eq. (1)), which means that the force-displacement is not exactly symmetric.

$$
R(x)=\rho_{1} x+\rho_{2} x^{2}+\rho_{3} x^{3}
$$

where the constants are in Table 1.

\subsection{Small amplitude vibrations}

The question arises, how accurate is the local tangent of $R(x)$, because it really depends on the order of the fit polynomial. Thus, local small amplitude measurement is carried out to determine the local "linear" dynamic behavior of the beam.

In this manner, we can define the local vibratory parameters such as natural frequency $\omega_{n}$, damping factor $\zeta$ and the local linear tangent $k$ of the stiffness characteristics $R(x)$. This can be determined by impact testing applying relatively small impulse excitation (peak force $F_{\max }<100 \mathrm{~N}$ ). However, excitation close to the point $\mathrm{B}$, that is in interest, is extremely difficult. Due to the flexibility of the structure, it is difficult to apply one distinct impact, without the back-swinging of low frequency modes causing multiple hits. This prevents good excitation over the frequency range of the first mode. To avoid this, a low resolution modal analysis was performed on the structure involving point B and E. The point $\mathrm{E}$ was chosen to avoid the assumingly node point at $\mathrm{A}$. This way the $H_{\mathrm{EE}}(\omega)$ and $H_{\mathrm{BE}}(\omega)$ frequency response functions (FRFs) in receptance were measured as

$$
\mathbf{H}(\omega)=\left[\begin{array}{c}
H_{\mathrm{EE}}(\omega) \\
H_{\mathrm{BE}}(\omega)
\end{array}\right]=\frac{Q \mathbf{p} v}{\omega \mathrm{i}-s}+\frac{\bar{Q} \overline{\mathbf{p}} \bar{v}}{\omega \mathrm{i}-\bar{s}},
$$

where the pole is defined as $s=-\omega_{\mathrm{n}} \zeta+\mathrm{i} \omega_{\mathrm{n}} \sqrt{1-\zeta^{2}}$. By using rational fraction polynomial (RFP) [12] method the spectral behavior $\left(\omega_{n}, \zeta\right)$ and the magnification behavior can be calculated by using the mode shapes $\mathbf{p}$, modal participation "vector" $v$ and the modal scaling factor $Q$. The parameters are listed in Table 2. With $Q$ and $p_{\mathrm{B}}$ the

Table 1 Force-displacement coefficients in Eq. (1).

\begin{tabular}{ccc}
\hline \multicolumn{3}{c}{ Table 1 Force-displacement coefficients in Eq. (1). } \\
\hline$\rho_{1}$ & $\rho_{2}$ & $\rho_{3}$ \\
\hline $18.72 \mathrm{~N} / \mathrm{mm}$ & $4.181 \mathrm{~N} / \mathrm{mm}^{2}$ & $4.528 \mathrm{~N} / \mathrm{mm}^{3}$ \\
\hline
\end{tabular}

reflected mass $m_{\mathrm{BB}}$ and stiffness $k_{\mathrm{BB}}$ between B excitation and B sensing points can be synthetized by using assumption for proportional damped system as

$k_{\mathrm{BB}}=-\frac{\omega_{\mathrm{n}}}{2 p_{\mathrm{B}}^{2} \sqrt{1-\zeta^{2}} \operatorname{Im} Q}$ and $k_{\mathrm{BB}}=m_{\mathrm{BB}} \omega_{\mathrm{n}}^{2}$,

then the synthesized FRF can have the following proportionally damped model as

$$
H_{\mathrm{BB}}(\omega)=\frac{1}{m_{\mathrm{BB}}} \frac{1}{-\omega^{2}+2 \zeta \omega_{\mathrm{n}} \omega \mathrm{i}+\omega_{\mathrm{n}}^{2}} .
$$

The reflected stiffness value $k_{\mathrm{BB}}$ refers to the linear coefficient $\rho_{1}$ of $R(x)$ in Eq. (1). We chose to accept $k_{\mathrm{BB}}$ instead of $\rho_{1}$ to describe local behavior, since the lowest the force the best is the determination of the local linear behavior. Moreover, the excitation of the impact spreads through the spectrum resulting in really slight excitation load in frequency domain. In this manner we performed a reduced order least square fitting for only the nonlinear coefficients. In Table 3 slight changes can be seen compared to the complete fitting since only $\rho_{2}$ and $\rho_{3}$ were the only unknowns in the least square fitting.

\subsection{Large amplitude vibrations}

In this section, we present two distinct large amplitude measurements. On the one hand, we used large sledge hammer to reach large amplitude oscillation in the nonlinear test-rig. On the other hand, we present a preloaded test, where we intended to avoid the incapability of hammer excitation for determining direct responses.

A response for impulse force triggered by sledge hammer blow is presented in Fig. 3 (a). Since the excitation at B-B is impossible due to the flexibility, we have excited the nonlinear beam at point $\mathrm{E}$ again. The use of the sledge hammer was necessary to achieve extremely large excitation load in a fairly stiff point and to have high amplitude response at $\mathrm{B}$. In this manner, acceptable low bound

Table 2 Measured local modal parameters of the first mode with

\begin{tabular}{ccc}
\multicolumn{3}{c}{$\|\mathrm{p}\|=1$ and $v=p_{\mathrm{E}}}$. \\
\hline$\omega_{\mathrm{n}}$ & $\zeta$ & $Q$ \\
\hline $52 \mathrm{~Hz}$ & $0.567 \%$ & $-8.1622 \mathrm{i} \mathrm{mm} / \mathrm{N} / \mathrm{s}$ \\
$\mathbf{p}=\left[\begin{array}{ll}p_{\mathrm{E}} & p_{\mathrm{B}}\end{array}\right]^{\mathrm{T}}$ & $m$ & $k$ \\
{$\left[\begin{array}{ll}0.083 & 0.996\end{array}\right]^{\mathrm{T}}$} & $0.196 \mathrm{~kg}$ & $20870.42 \mathrm{~N} / \mathrm{m}$ \\
\hline
\end{tabular}

Table 3 Force-displacement coefficients with corrected local parameters.

\begin{tabular}{ccc}
\hline$\rho_{1}=k_{\mathrm{BB}}$ & $\rho_{2}$ & $\rho_{3}$ \\
\hline $20.18 \mathrm{~N} / \mathrm{mm}$ & $4.105 \mathrm{~N} / \mathrm{mm}^{2}$ & $4.336 \mathrm{~N} / \mathrm{mm}^{3}$ \\
\hline
\end{tabular}


a)

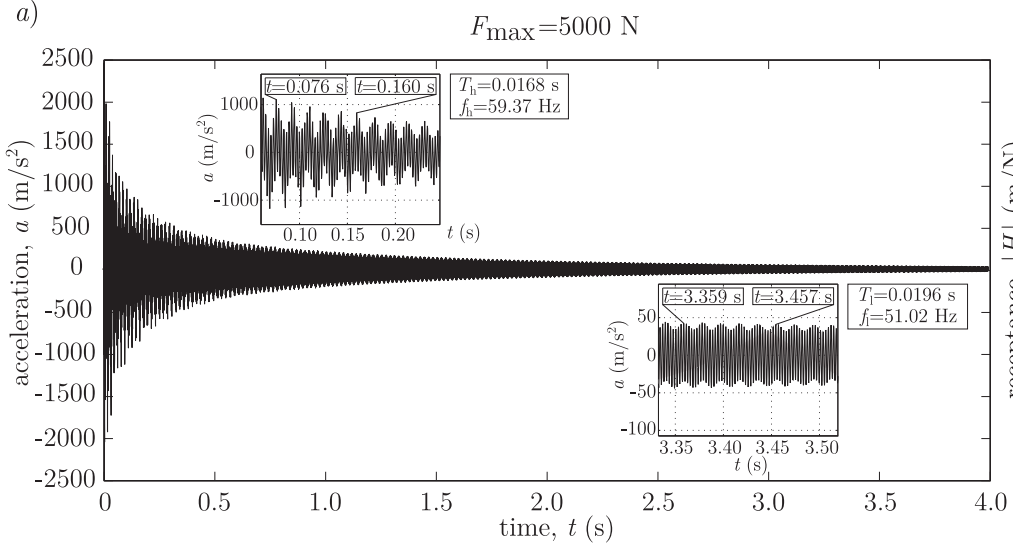

b)

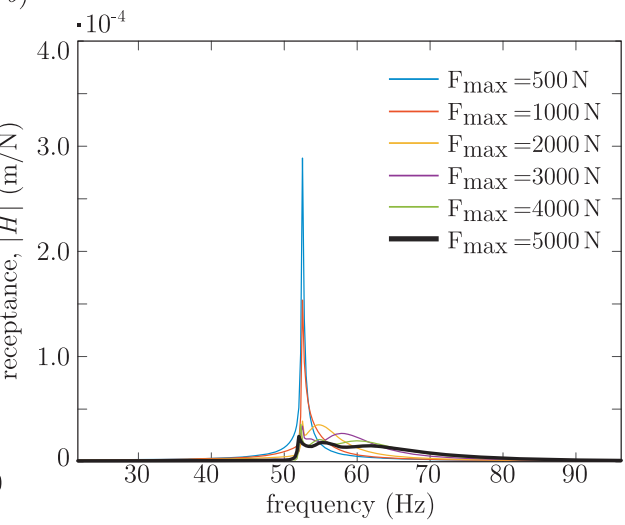

Fig. 3 (a) represents response at point B for the large blow by sledge hammer at point $\mathrm{E}$ and presents the frequency change for high $\left(T_{\mathrm{h}}, f_{\mathrm{h}}\right)$ and for low $\left(T_{1}, f_{1}\right)$ amplitude regions. Panel (b) shows the quasi-"FRF"s for various impulse excitation.

excitation and its good coherence were ensured providing, methodology-wise good results. The evaluated "FRFs"s for different force levels $\left(F_{\text {max }, t}\right)$ are presented in Fig. 3 (b). Mathematically speaking these functions are not applicable as FRF's, they are not representing, in any manner, linear and/or nonlinear behavior. This means, these quasi-"FRF"s can only present the appearance of nonlinearities if it is recognizable at all.

On the response presented in Fig. 3 (a) one can easily trace the varying frequencies of the first mode w.r.t. the vibration amplitude. It shows stiffening characteristics with higher frequencies for larger amplitudes. By compiling the naive quasi-"FRF"s presented in Fig. 3 (b), one can see first moderate, then extreme drop in the main resonance peak. The larger excitation force spreads the response across the spectrum resulting in flat and strange ("smeared") resonance, which clearly is the sign of higher frequency content in the decaying nonlinear response.

Because of the inadequate methodology of modal analysis for large amplitude nonlinear vibration and the impossible sense of direct hits at B-B, we decided to perform free vibratory test with initial preload. The preload was given similarly as in the static characteristic tests by simply using rope and hanging mass. This way the measurement is repeatable, and the free vibratory behavior appears in the decaying motion of point $\mathrm{B}$. Later this measurement can be compared with results simulated by using the B-B static characteristics in Eq. (1).

Since the structure has a small amount of dissipation, the amplitude decreases over fairly long time. For this preload triggered free vibration we expect to see a change in the frequency as well, similar to the presented backbone curve. To show this, we divide the signal into shorter time windows. If we take the discrete Fourier transform (DFT) of these individual signal sections, we can find a peak around the first natural frequency, which is considered as the instantaneous frequency of the section. However, for $\Delta f=1 \mathrm{~Hz}$ frequency steps in the DFT, we would need to take $T=1 / \Delta f=1 \mathrm{~s}$ time sections due to the Fourier uncertainty. But, the measured data is around $1 \mathrm{~s}$ long, which cannot be divided into further sections. Moreover, if we want to achieve smaller frequency steps (higher precision), the time sections had to be even longer, so it means that this method cannot be used to get accurate results.

Another approach to this problem includes the use of a digital filter. The vibrations of the first mode can be separated with a low pass filter (Fig. 4 (a)).

The amplitude and the period of the filtered signal then can be read easily. The cut-off frequency is $f_{\mathrm{c}}=200 \mathrm{~Hz}$, as we expect the frequency of the vibration to be roughly around the first natural frequency. The normalized cut-off frequency is

$\phi_{\mathrm{c}}=\frac{f_{\mathrm{c}}}{f_{\mathrm{s}} / 2}=0.049$,

which is a quite small value, as we used a large, $f_{\mathrm{s}}=8192 \mathrm{~Hz}$ sampling rate. As seen on Fig. 4 (a) the lower frequency components pass without distortion in the amplitude, but the phase shift is significant. To avoid this problem, the signal is filtered in both the forward and reverse directions, which yields a zero-phase filtered signal.

Fig. 4 (b) shows the acceleration signal of the first mode only. We can clearly distinguish each period of the signal, so the amplitude and the time period can be read systematically, as seen on Fig. 4 (c), (d). 

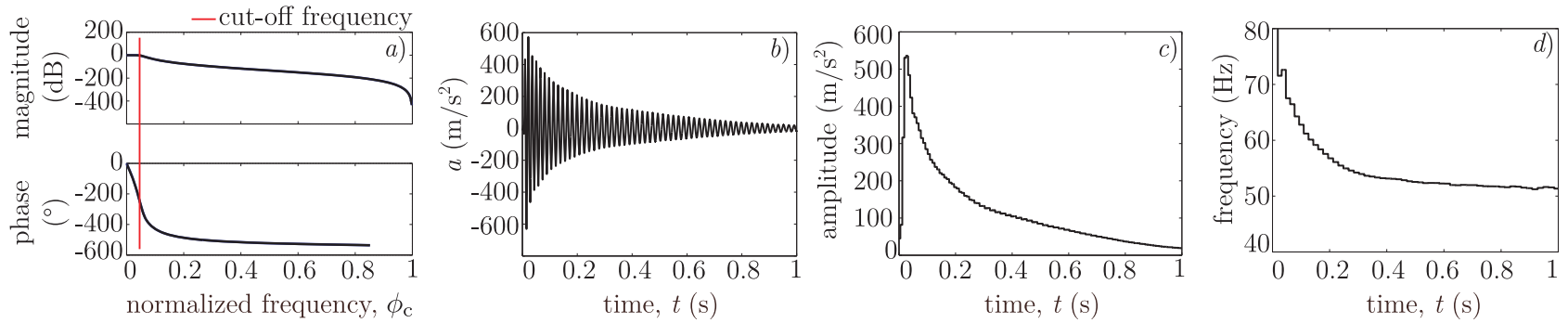

Fig. 4 (a) presents the low pass filter used, while (b) shows the filtered free vibratory motion after using constant preload. The (c) and (d) show the extracted amplitude and the corresponding frequency over time.

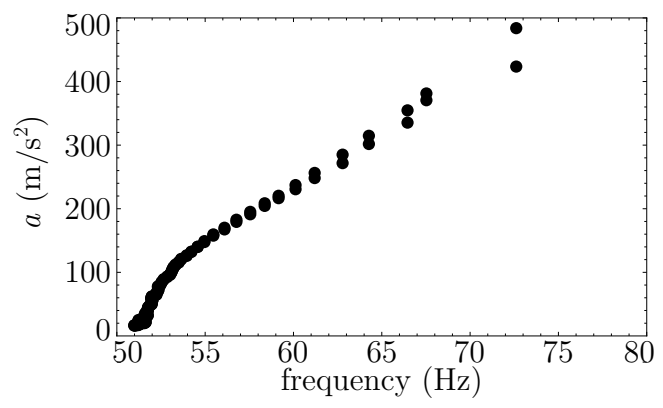

Fig. 5 The acceleration amplitude-frequency of the filtered signal.

By using relations depicted in Fig. 4 (c), (d) one can plot free vibratory path in the frequency-amplitude diagram (Fig. 5), actually formulating the measured backbone curve. Note that, linear system would be attenuated along a "vertical" line on this plot ending also at the (local) natural frequency. The plot clearly resembles the stiffening nonlinear response resonance shown in the sketch Fig. 1, although for convenience the acceleration amplitude was used here w.r.t. the momentary frequency.

In order to compare the results with the theory, the aim is to plot the result related to the amplitude dependency in receptance frequency response function fashion. Although, the multiplicative purpose of this nonlinear receptance response with the excitation force cannot be applied in this nonlinear case. In Fig. 5 one can realize two things. On the one hand, the expected behavior was measured, namely, small amplitude vibration has the "linear" natural frequency $f_{\mathrm{n}}$ taken from the end of the free vibratory signal (see Fig. 4 (b)). On the other hand, the peak-to-peak amplitudes do not seem to be symmetric, which can be the result of the asymmetry in the static stiffness measurement (see Fig. 2 (c)).

\section{Analytical approach}

There are some methods to approximate the amplitude dependency of the transient behavior of one DoF nonlinear mechanical oscillator. However, there is no exact method to derive an explicit closed form for the free vibratory motion for this system.

To reach the asymmetry recognized in Fig. 2 (c) the second order monomial is also included in the equation of motion, here:

$\ddot{x}(t)+2 \zeta \omega_{\mathrm{n}} \dot{x}(t)+\omega_{\mathrm{n}}^{2} x(t)+\mu_{2} x^{2}(t)+\mu_{3} x^{3}(t)=0$,

where $\mu_{2}=\rho_{2} / m$ and $\mu_{3}=\rho_{3} / m$ ( $m:=m_{\mathrm{BB}}$, see Eq. (3)) respectively.

We use one form of asymptotic analysis method, namely, the Ljapunov-Poincaré method for periodic motion [13]. In order to ensure the applicability of the method to calculate the vibration period $T=2 / \beta$, we consider undamped version of Eq. (6), where the actual angular vibration frequency is given as the function of the nonlinear parameter $\mu=\mu_{1} / \varepsilon_{1}=\mu_{2} / \varepsilon_{2}$ as

$\beta^{2}:=\omega_{\mathrm{n}}{ }^{2}+\mu h_{1}+\mu^{2} h_{2}+\ldots$.

In Eq. (7), $\varepsilon_{1}$ and $\varepsilon_{2}$ parameters are chosen appropriately. The basic idea of the calculation is to determine the time-periodic solution $x(t)=x(t+T)$ as a series of the nonlinearity with

$x(t):=\sum_{j=0}^{\infty} \mu^{j} x_{j}(t)$

Here each $j$ th member of Eq. (8) satisfies the boundary condition $x_{j}(t)=x_{j}(t+T)$ and by vanishing the internal resonances the $h_{k}$ 's $(k \geq 1)$ can be determined using the initial conditions $x(0)=x_{0}(0):=A$ and $x_{j}(0):=0(j>1)$ and $\dot{x_{j}}(0):=0$ (all $\left.j\right)$ for the undamped $(\zeta=0)$ system Eq. (6). Internal resonances appears on the separated $\mathcal{O}\left(\varepsilon^{k}\right)$ equations as a resonant excitation, which provides essentially nonperiodic partial solutions $x_{j}$ Eq. (8), which have to be vanished by equating $h_{j}$ 's in Eq. (7).

In Fig. 6 the results for cubic fitting is presented using data of Table 3. The analytical amplitude-frequency curves 


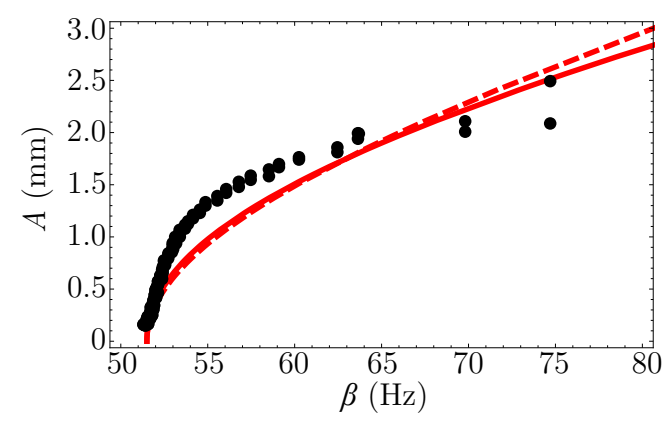

Fig. 6 The backbone curve determined by simple 3rd order equation of motion Eq. (6) by using 3rd order polynomial fitting as in Fig. 2 (c). Dashed curve related to $\beta\left(h_{1}\right)$, while continuous to $\beta\left(h_{1}, h_{2}\right)$ (see Eq. (7)).

determined with the first $\beta\left(h_{1}\right)$ and with the combined first and second $\beta\left(h_{1}, h_{2}\right)$ internal resonances do not really fit on the measured ones. The empirical backbone curve has larger curvature, which allows to conclude higher orders of the stiffness nonlinearity play an important role.

In this manner higher order fittings (see Fig. 7 (a), (c)) were performed, with which the entire calculation can be repeated. The equation of motion assumed in the form

$\ddot{x}(t)+2 \zeta \omega_{\mathrm{n}} \dot{x}(t)+\omega_{\mathrm{n}}{ }^{2} x(t)+\sum_{k=1}^{p} \mu_{k} x^{k}(t)=0$,

where $p$ is the order of the polynomial fitting and $\mu_{k}=\mu \varepsilon_{k}$, accordingly. Without presenting the partial steps, including the first order internal resonance we can derive the following for the vibration frequency

$$
\beta\left(h_{1}(A)\right)=\sqrt{\omega_{\mathrm{n}}{ }^{2}+\mu\left(\varepsilon_{3} \frac{3 A^{2}}{4}+\varepsilon_{5} \frac{10 A^{4}}{16}+\varepsilon_{7} \frac{35 A^{6}}{64}\right)} .
$$

While taking into account the second internal resonance the following long form is obtained:
Table 4 Coefficients of the fit force-displacement curves (see Fig. 8)

\begin{tabular}{cccc}
\hline$\rho_{i}\left(\mathrm{~N} / \mathrm{mm}^{i}\right)$ & 5 th & 7 th & 11 th \\
\hline$\rho_{1}:=k$ & 20.18 & 20.18 & 20.18 \\
$\rho_{2}$ & 6.251 & 8.365 & 8.863 \\
$\rho_{3}$ & 4.584 & 2.764 & 2.117 \\
$\rho_{4}$ & -0.306 & -0.821 & -3.266 \\
$\rho_{5}$ & -0.048 & 0.408 & 0.257 \\
$\rho_{6}$ & - & 0.028 & 0.014 \\
$\rho_{7}$ & - & -0.026 & 0.217 \\
$\rho_{8}$ & - & - & -0.240 \\
$\rho_{9}$ & - & - & -0.053 \\
$\rho_{10}$ & - & - & 0.014 \\
$\rho_{11}$ & - & - & 0.033 \\
\hline
\end{tabular}

second internal resonances related to Eq. (10) and Eq. (11), respectively. The 7 th order polynomial fitting (Fig. 7 (d)) with both internal resonances follows the measurement well. A tiny fold can be realized on the calculated parametric curve, that suggests that the analytical formalism allows, actually, to predict different local behavior. The measurement does not show this property, but in the higher frequency zone the growth is captured better.

Surely, there is a convergence by increasing the order of the polynomial fitting (see Table 4), however, there is an arithmetical limit for this analytical methodology. Due to the large amount of measurement points in the static characteristics, few other larger order fittings can be realized in order to see convergence on the backbone curves. This is checked in the next section, where we determine these backbone curves numerically, without dealing with internal resonances supposingly serving more accurate and convergent solutions.

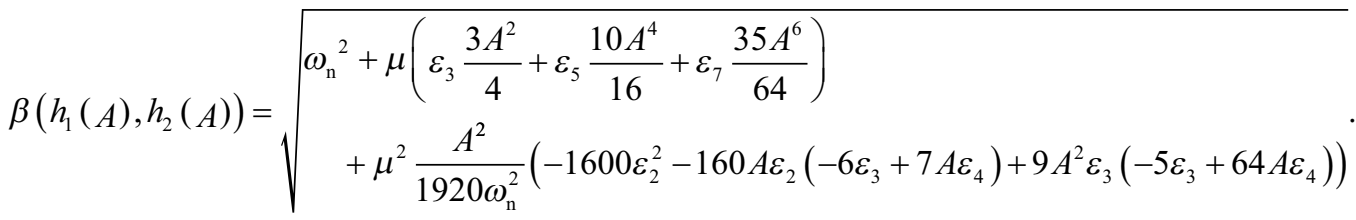

Higher order fittings were performed (see Table 4) by keeping the local behavior with the determined linear stiffness $k_{\mathrm{BB}}$ from Eq. (3). In Fig. 7 (a)-(c) one can realize that the fittings still look adequate, but their coefficients change with convergence in low order coefficients (see Table 4). This obviously has an effect on the backbone curves. In Fig. 7 (b)-(d) the corresponding backbone curves are presented for first and combined first and
If we compare the analytical and the measurement results (Fig. 7 (b)-(d)), we can see that the linear (small amplitude vibration) frequencies are slightly different $(1 \mathrm{~Hz})$. This is likely the result of the fact that, between the linear and large amplitude measurements, the bolt preloads in the fixations were slightly modified. Aside of this, the measurement data and the analytical solution are quite similar, especially for the higher order static force-displacement approximation. 
a)
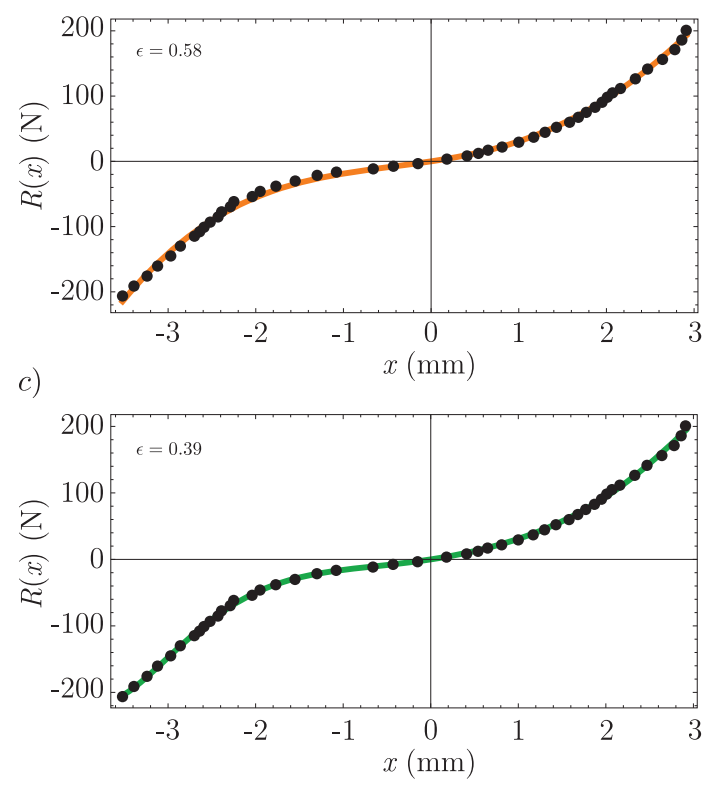

b)
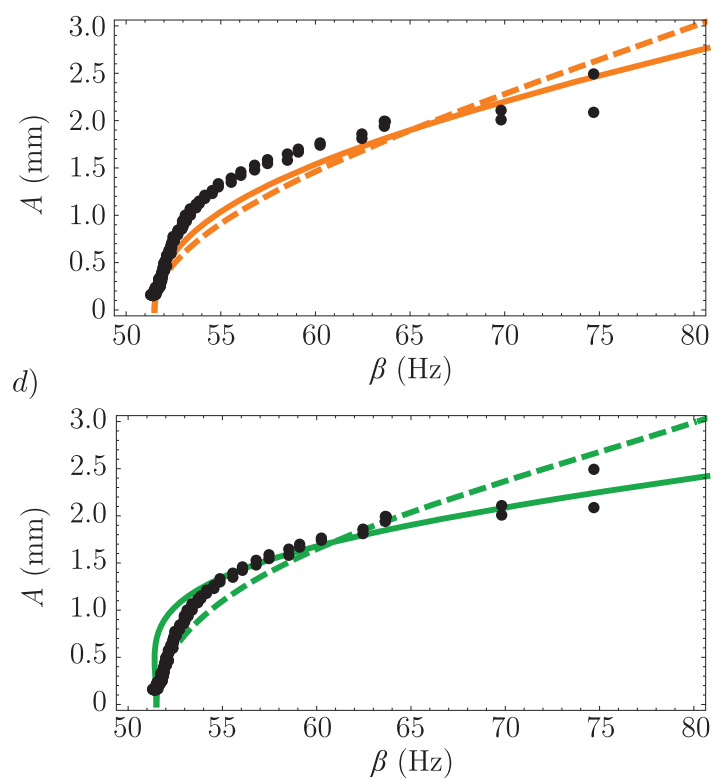

Fig. 7 In (a) the actual 5th and 7th fitting are presented, while (b) shows the corresponding amplitude-frequency curves determined only for the first $\beta\left(h_{1}\right)$ (dashed) and for the combined first and second order internal resonances $\beta\left(h_{1}, h_{2}\right)$ (continuous).

\section{Numerical Approach}

Free vibratory response behavior is characterized with backbone curves, along which the mechanical system settles down, slowly reaching zero amplitude oscillation with $\omega_{\mathrm{n}}$ natural frequency. It is essential to have a numerical method, with which the behavior of the free vibratory system can be characterized to avoid performing vast amount of time domain simulations with various initial conditions. Moreover, by using a general numerical method higher order approximation can be easily checked. Also, backbone curves are referring to the shape of the nonlinear harmonic response behavior, that can be important to determine stationary behavior of machines including nonlinearities.

The method presented in this part is based on continuation algorithms like AUTO [14], MATCONT [15], PDDE-CONT, KNUT [16] and DDE-BIFTOOL [17]. These algorithms are capable of discretizing deterministic dynamical systems and trace special solutions in the phase space w.r.t. a given parameter. In this way, the behavior of the dynamics can be mapped through special objects in the phase space like equilibrium, periodic or heteroclinic orbits. To compile backbone curves a natural choice is to trace the undamped system for a given initial amplitude as a numerical counterpart of the asymptotic method described in the previous section.

If the system is undamped closed orbits with a certain time period $T$ are formed in the phase space. The above mentioned algorithms first rescale the system with the period $T$ and then apply an interpolation scheme usually based on Lagrange polynomials. By describing the periodic orbit with a concatenated segmented solution, sampling at Legendre points, the dynamic system is projected to a set of impulse functions forming nonlinear algebraic system. This system for autonomous systems are not complete due to the time invariance. To release this problem Poincaré phase condition can be used [17]. However, to find backbone curves the phase condition is replaced with a condition to select the amplitude in interest.

Simply the condition can be given in the form for peakto-peak amplitude:

$\max _{t=0}^{T} x(t)-\min _{t=0}^{T} x(t)-2 A=0$

Although, its derivatives w.r.t. all state derivatives and parameters have to be given to the algorithms to deliver efficiently and accurately solutions with Newton-Raphson method.

\section{Conclusion}

The result of this work has two folds. On one hand, we showed that the resemblance of the theoretically incorrect FRF (quasi-FRF measured with impulse excitation) to its harmonic response does not have any theoretical or practical relevance. The seemingly steep edge (e.g. thick black in Fig. 3 (b)) does not reveal a hysteretic behavior, it is 
a)

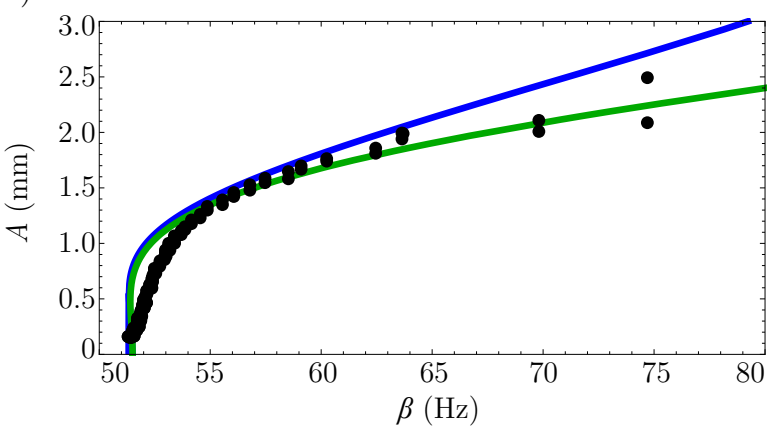

b)

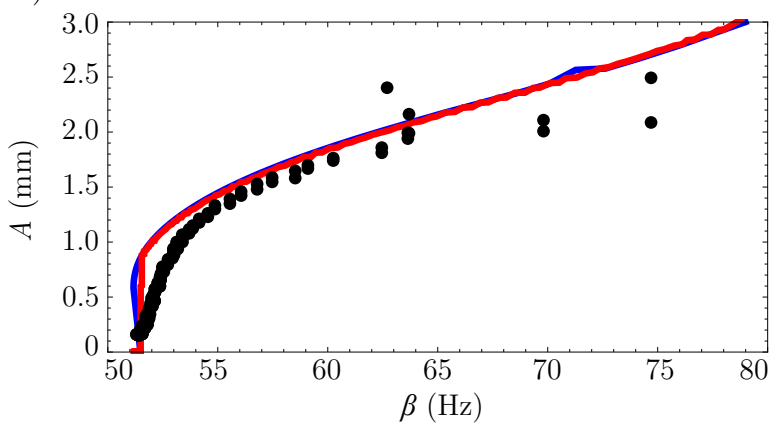

Fig. 8 (a) shows the comparison between the 7th order analytical approximation (green) and the solution with the continuation method (blue) for the same order, while (b) shows time simulation (red) and continuation solution (blue) for 11th order.

simply showing the energy content of different frequency components, which for an ideal hardening characteristic cannot be (theoretically) less than the natural frequency. This result has a practical reflection because quasi-FRFs of a nonlinear mechanical systems are often cited as following the hysteretic behavior of the anyway harmonically excited nonlinear stationary response (Fig. 1), which statement is clearly incorrect.

On the other hand, we showed by analytical asymptotic method, that inadequate fitting of continuous nonlinearities can bring in large unexpected error in amplitude prediction. The higher order terms determine the curvature of the backbone curves. In this manner, it is essential to have good fitting for accurate amplitude prediction over large bandwidth.

Apart from this, we have compared our analytical prediction with continuation results to find the convergent solution

\section{References}

[1] Ahlquist, J. R., Carreño, J. M., Climent, H. "Assessment of Nonlinear Structural Response in A400M GVT", In: $28^{\text {th }}$ International Modal Analysis Conference (IMAC), Jacksonville, Florida, USA, 2010. [online] Available at: http://www.am.chalmers.se/ thab/IMAC/2010/PDFs/Papers/s45p003.pdf [Accessed: 30 April 2018]

[2] Bechly, M. E., Clausen, P. D. "Structural design of a composite wind turbine blade using finite element analysis", Computers \& Structures, 63(3), pp. 639-646, 1997.

https://doi.org/10.1016/S0045-7949(96)00387-2

[3] Hanna, N. H., Tobias, S. A. "A Theory of Nonlinear Regenerative Chatter", Journal of Engineering for Industry, 96(1), pp. 247-255, 1974.

https://doi.org/10.1115/1.3438305

[4] Nayfeh, A. H., Chin, C.-M., Pratt, J. "Perturbation Methods in Nonlinear Dynamics - Applications to Machining Dynamics", Journal of Manufacturing Science and Engineering, 119(4A), pp. 485-493, 1997.

https://doi.org/10.1115/1.2831178
(Fig. 8). The continuation was performed on the undamped system using a special condition for the peak-to-peak amplitude replacing the usual Poincaré phase condition.

In summary, the measurement showed that the nonlinear "modal" description has many questionable modeling issues that needs analysis on its foundation. The work showed that the natural choice of polynomial describing function of the stiffness characteristics can introduce large errors in the amplitude prediction even with high order fitting. A future methodology should be independent of these modeling errors.

\section{Acknowledgement}

Supported by the Hungarian EMMI ÚNKP-17-4-III New National Excellence Program of the Ministry of Human Capacities.

[5] Kerschen, G., Peeters, M., Golinval, J. V., Vakakis, A. F. "Nonlinear normal modes, Part I: A useful framework for the structural dynamicist", Mechanical Systems and Signal Processing, 23(1), pp. 170-194, 2009.

https://doi.org/10.1016/j.ymssp.2008.04.002

[6] Tseng, W.-Y., Dugundji, J. "Nonlinear Vibrations of a Buckled Beam Under Harmonic Excitation", Journal of Applied Mechanics, 38(2), pp. 467-476, 1971.

https://doi.org/10.1115/1.3408799

[7] Hill, T. L., Cammarano, A., Neild, S. A., Wagg, D. J. "Interpreting the forced responses of a two-degree-of-freedom nonlinear oscillator using backbone curves", Journal of Sound and Vibration, 349, pp. 276-288, 2015.

https://doi.org/10.1016/j.jsv.2015.03.030

[8] Rosenberg, R. M. "Normal Modes of Nonlinear Dual-Mode Systems", Journal of Applied Mechanics, 27(2), pp. 263-268, 1960. https://doi.org/10.1115/1.3643948

[9] Shaw, S., Pierre, C. "Non-linear normal modes and invariant manifolds", Journal of Sound and Vibration, 150(1), pp. 170-173, 1991. https://doi.org/10.1016/0022-460X(91)90412-D 
[10] Szalai, R., Ehrhardt, D., Haller, G. "Nonlinear model identifcation and spectral submanifolds for multidegree-of-freedom mechanical vibrations", Proceedings of the Royal Society A: Mathematical, Physical and Engineering Science, 473(2202), pp. 1-19, 2017. https://doi.org/10.1098/rspa.2016.0759

[11] Kerschen, G., Worden, K., Vakakis, A. F., Golinval, J.-C. "Past, present and future of nonlinear system identification in structural dynamics", Mechanical Systems and Signal Processing, 20(3), pp. 505-592, 2006. https://doi.org/10.1016/j.ymssp.2005.04.008

[12] Richardson, M. H., Formenti, D. L. "Parameter Estimation from Frequency Response Measurements Using Rational Fraction Polynomials", presented at 1st IMAC Conference, Orlando, FL, USA, Nov. 8-10, 1982.

[13] Tkhai, V. N. "The Lyapunov-Poincaré method in the theory of periodic motions", Journal of Applied Mathematics and Mechanics, 62(3), pp. 325-339, 1998.

https://doi.org/10.1016/S0021-8928(98)00044-6
[14] Doedel, E., Champneys, A., Fairgrieve, T., Kuznetsov, Y., Sandstede, B., Wang, X. "AUTO 97: continuation and bifurcation software for ordinary differential equations", 1997. [online] Available at: http://indy.cs.concordia.ca/auto/ [Accessed: 30 April 2018]

[15] Dhooge, A., Govaerts, W., Kuznetsov, Y. A., Mestrom, W., Riet, A. M., Sautois, B. "MATCONT and CL_MATCONT: Continuation toolboxes in Matlab", Universiteit Gent, Belgium, Utrecht University, The Netherlands, 2006.

[16] Szalai, R. "Knut: a continuation and bifurcation software for delay-differential equations", 2013. [online] Available at: http:// rs1909.github.io/knut/ [Accessed: 30 April 2018]

[17] Engelborghs, K., Luzyanina, T., Samaey, G. "DDE-BIFTOOL V. 2.00: A Matlab package for bifurcation analysis of delay differential equations", Department of Computer Science, K.U. Leuven, Leuven, Belgium, Rep. TW330, 2001. 